\title{
Theory of Carrier Phase Ambiguity Resolution
}

\section{P. J. G. Teunissen}

Department of Mathematical Geodesy and Positioning, Delft University of Technology, Thijsseweg 11, 2629 J A Delft, The Netherlands

\begin{abstract}
Carrier phase ambiguity resolution is the key to high precision Global Navigation Satellite System (GNSS) positioning and navigation. It applies to a great varicty of current and future models of GPS, modernized GPS and Galileo. A proper handling of carrier phase ambiguity resolution requires a proper understanding of the underlying theory of integer inference. In this contribution a brief review is given of the probabilistic theory of integer ambiguity estimation. We describe the concept of ambiguity pull-in regions, introduce the class of admissible integer estimators, determine their probability mass functions and show how their variability affect the uncertainty in the so-called 'fixed' bascline solution. The theory is worked out in morc detail for integer least-squares and integer bootstrapping. It is shown that the integer least-squares principle maximizes the probability of correct integer estimation. Sharp and easy-to-compute bounds are given for both the ambiguity success rate and the baseline's probability of concentration. Finally the probability density function of the ambiguity residuals is determined. This allows one for the first time to formulate rigorous tests for the integerness of the parameters.
\end{abstract}

Key words: GNSS; ambiguity resolution; integer least-squares

CLC number: P 228.4

Received date: 2002-08-24

Biography: P.J.G. Teunissen (1957-), mals, Professor, Pin.D, research direction: GNSS data processing strategies for medium scaled networks with an emphasis on ambiguity resolution. E-mail: P.J.G.Teunissen@citg.tudelft.nl

\section{Introduction}

G lobal Navigation Satellite System (GNSS) ambiguity resolution is the process of resolving the unknown cycle ambiguities of double difference (DD) carrier phase data as integers. It is the key to high precision GNSS positioning and navigation. The availability of a theory of integer inference is therefore a prerequisite for a proper handling and understanding of the various intricate aspects of carrier phase ambiguity resolution. Although the theory of integer inference as a whole is unfortunately still far from mature, significant progress has been made in the last decade in the area of integer ambiguity estimation. This holds true for the computational aspects of integer estimation as well as for the corresponding probabilistic aspects. We now have a theoretical framework available which enables one to define integer estimators unequivocally, to compare their performance, to single-out optimal estimators, to judge the probabilistic consequences for the so-called 'fixed' baseline solution and to design measurement set-ups in accordance to specifications. In addition the framework has also enabled one to identify pitfalls in some of the earlier proposed methods of ambiguity resolution. It is the purpose of the current invited contribution to give a brief review of this probabilistic framework of integer estimation. The focus will be on integer least-squares estimation and some closely related integer estimation principles. The presentation will be non-Bayesian throughout. For a Bayesian approach to ambiguity resolution we refer to e.g. Ref.[1-3]. The presentation will also not touch upon the theory of integer validation, a theory which unfortunately is still in its infancy. But some first results, as reported in Ref.[3,4], will be given.

The practical importance of carrier phase ambiguity resolution becomes clear when one realizes the great variety of current and future GNSS models to which it applies. These models may differ greatly in complexity and diversity. They 
range from single-baseline models used for kinematic positioning to multi-baseline models used as a tool for studying geodynamic phenomena. The models may or may not have the relative receiver-satellite geometry included. They may also be discriminated as to whether the slave receiver(s) are stationary or in motion. When in motion, one solves for one or more trajectories, since with the receiver-satellite geometry included, one will have new coordinate unknowns for each epoch. One may also discriminate between the models as to whether or not the differential atmospheric delays (ionosphere and troposphere) are included as unknowns. In the case of sufficiently short baselines they are usually excluded.

Apart from the current Global Positioning System (GPS) models, carrier phase ambiguity resolution also applies to the future modernized GPS and the future European Galileo GNSS. An overview of GNSS models, together with their applications in surveying, navigation, geodesy and geophysics, can be found in textbooks such as Ref.[6-11].

In the present contribution we emphasize the probabilistic aspects of integer ambiguity estimation. This contribution is organized as follows. In Section 1 we introduce a general class of integer ambiguity estimators, determine their probability mass functions and show how their variability affect the uncertainty in the computed GNSS baselines. This theory is worked out in Sections 2 and 3 for two of the most important integer ambiguity estimators. We refrain from giving proofs of the theorems and corollaries. For these proofs we refer to the referenced literature. In Section 2 we discuss the properties of integer bootstrapping and in Section 3 those of integer least-squares. The properties of these two estimators are compared. It is shown that integer least-squares maximizes the probability of correct integer estimation. We also give sharp and easy-to-compute bounds for the ambiguity success rate. In the final Section 4 we present the joint pdf of the 'float' and 'fixed' ambiguities and from it determine the pdf of the ambiguity residuals.

\section{Integer Ambiguity Resolution}

\subsection{The GNSS Model}

As our point of departure we will take the following system of linear(ized) observation equations

$$
y=A a+B b+e
$$

where $y$ is the given GNSS data vector of order $m, a$ and $b$ are the unknown parameter vectors respectively of order $n$ and $p$, and where $e$ is the noise vector, $A$ and $B$ are the corresponding design matrices. In principle all the GNSS models can be cast in this frame of observation equations. The data vector $y$ will usually consist of the 'observed minus computed' singleor dual-frequency double-difference (DD) phase and/or pseudorange (code) observations accumulated over all observation epochs. The entries of vector $a$ are then the DD carrier phase ambiguities, expressed in units of cycles rather than range. They are known to be integers, $a \in Z^{n}$. The entries of the vector $b$ will consist of the remaining unknown parameters, such as for instance baseline components (coordinates) and possibly atmospheric delay parameters (troposphere, ionosphere). They are known to be real-valued, $b \in R^{p}$.

The procedure which is usually followed for solving the GNSS model (1), can be divided into three steps. In the first step one simply disregards the integer constraints a $a \in Z^{n}$ on the ambiguities and performs a standard least-squares adjustment. As a result one obtains the (real-valued) estimates of $a$ and $b$, together with their variance-covariance (vc-) matrix

$$
\left[\begin{array}{c}
\hat{a} \\
\hat{b}
\end{array}\right], \quad\left[\begin{array}{cc}
Q_{\hat{a}} & Q_{\hat{a} \hat{b}} \\
Q_{\hat{b} \hat{a}} & Q_{\hat{b}}
\end{array}\right]
$$

This solution is referred to as the 'float' solution. In the second step the 'float' ambiguity estimate $\hat{a}$ is used to compute the corresponding integer ambiguity estimate $\vec{a}$. This implies that a mapping $S: R^{n} \mapsto Z^{n}$, from the $n$-dimensional space of reals to the $n$-dimensional space of integers, is introduced such that

$$
\breve{a}=S(\hat{a})
$$

Once the integer ambiguities are computed, they are used in the third step to finally correct the 'float' estimate of $b$. As a result one obtains the 'fixed' solution

$$
\breve{b}=\hat{b}-Q_{\hat{b} \hat{a}} Q_{\hat{a}}^{-1}(\hat{a}-\breve{a})
$$

In the present review we will primarily focus our attention on the probabilistic properties of Eq.(3) and Eq.(4).

\subsection{Admissible Integer Estimation}

There are many ways of computing an integer ambiguity vector $\vec{a}$ from its real-valued counterpart $\hat{a}$ To each such method belongs a mapping $S: R^{n} \mapsto Z^{n}$ from the $n$-dimensional space of real numbers to the $n$-dimensional space of integers. Due to the discrete nature of $Z^{n}$, the map $S$ will not be one-to-one, but instead a many-to-one map. This implies that different real-valued ambiguity vectors will be mapped to the 
same integer vector. One can therefore assign a subset $S_{z} \subset R^{n}$ to each integer vector $z \in Z^{n}$ :

$$
S_{z}=\left\{x \in R^{n} \mid z=S(x)\right\}, z \in Z^{n}
$$

The subset $S_{z}$ contains all real-valued ambiguity vectors that will be mapped by $S$ to the same integer vector $z \in Z^{n}$. This subset is referred to as the pull-in region of $z$ (see Fig.1). It is the region in which all ambiguity 'float' solutions are pulled to the same 'float' ambiguity vector $z$.

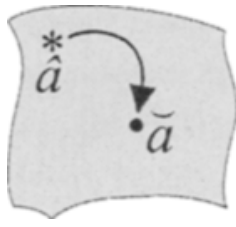

Fig.1 An ambiguity pull-in region of $z=\breve{a}$

Using the pull-in regions, one can give an explicit expression for the corresponding integer ambiguity estimator. It reads

$$
\breve{a}=\sum_{z \in Z^{n}} z s_{z}(\hat{a})
$$

with the indicator function:

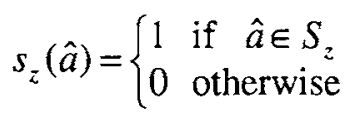

Since the pull-in regions define the integer estimator completely, one can define classes of integer estimators by imposing various conditions on the pull-in regions. One such class is referred to as the class of admissible integer estimators. This class was introduced in Ref.[12] and it is defined as follows.

Definition 1 The integer estimator $\breve{a}=\sum_{z \in Z^{n}} z s_{z}(\hat{a})$ is said to be admissible if

1) $\bigcup_{i \in z^{n}} S_{z}=R^{n}$

2) $\operatorname{Int}\left(S_{z_{1}}\right) \cap \operatorname{Int}\left(S_{z_{2}}\right) \notin 0, \forall z_{1}, z_{2} \in Z^{n}, z_{1} \neq z_{2}$

3) $S_{z}=z+S_{0}, \forall z \in Z^{n}$

This definition is motivated as follows (see Fig.2). Each one of the above three conditions describes a property of which it seems reasonable that it is possessed by an arbitrary integer ambiguity estimator. The first condition states that the pull in regions should not leave any gaps and the second that they should not overlap. The absence of gaps is needed in order to be able to map any 'float' solution $\hat{a} \in R^{n}$ to $Z^{n}$, while the absence of overlaps is needed to guarantee that the 'float' solution is mapped to just one integer vector. Note that we allow the pull-in regions to have common boundaries.
This is permitted if we assume to have zero probability that $\hat{a}$ lies on one of the boundaries. This will be the case when the probability density function (pdf) of $\hat{a}$ is continuous.

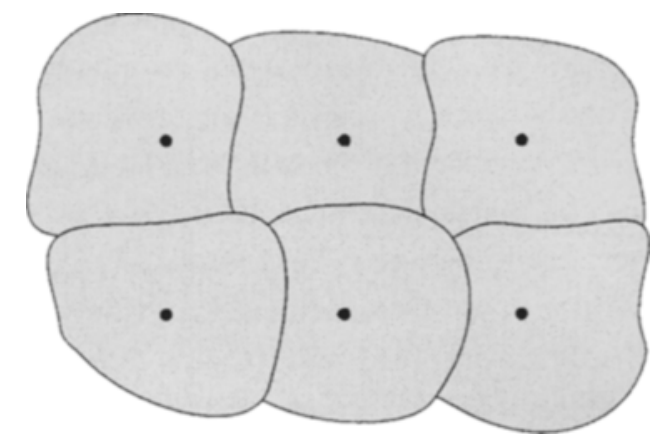

Fig.2 Pull-in regions that $\operatorname{cover} \boldsymbol{R}^{n}$ without gaps and overlaps

The third and last condition of the definition follows from the requirement that $S(x+z)=S(x)+z, \forall x \in R^{n}$, $z \in Z^{n}$ (see Fig.3). Also this condition is a reasonable one to ask for. It states that when the 'float' solution is perturbed by $z \in Z^{n}$, the corresponding integer solution is perturbed by the same amount. This property allows one to apply the integer remove-restore technique: $S(\hat{a}-z)+z=S(\hat{a})$. It therefore allows one to work with the fractional parts of the entries of $\hat{a}$, instead of with its complete entries.

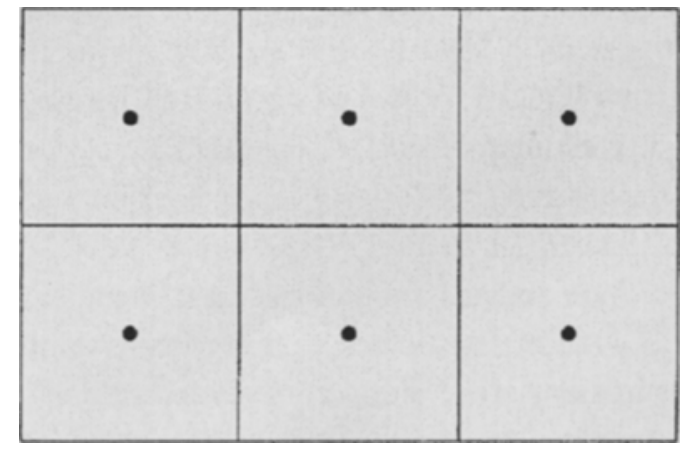

Fig.3 An example of integer translational invariant pull-in regions that $\operatorname{cover} \boldsymbol{R}^{n}$ without gaps and overlaps

With the division of $R^{n}$ into mutually exclusive pull-in regions, we are now in the position to consider the distribution of $\bar{a}$. This distribution is of the discrete type and it will be denoted as $P(\breve{a}=z)$. It is a probability mass function, having zero masses at non-grid points and nonzero masses at some or all grid points. If we denote the continuous probability density function of $\hat{a}$ as $p_{\hat{a}}(x)$, the distribution of $\breve{a}$ follows as

$$
P(\check{a}=z)=\int_{S_{z}} p_{\hat{a}}(x) \mathrm{d} x, \quad z \in Z^{n}
$$


This expression holds for any distribution the 'float' ambiguities $\hat{a}$ might have. In most GNSS applications however, one assumes the vector of observables $y$ to be normally distributed. The estimator $\hat{a}$ is therefore normally distributed too, with mean $a \in Z^{n}$ and vc-matrix $Q_{\hat{a}}$. Its probability density function reads

$$
p_{\hat{a}}(x)=\frac{1}{\sqrt{\operatorname{det}\left(Q_{\hat{a}}\right)}(2 \pi)^{\frac{1}{2} n}} \exp \left\{-\frac{1}{2}\|x-a\|_{Q_{\hat{a}}^{\frac{2}{a}}}\right\}
$$

with the squared weighted norm $\|\cdot\|_{Q_{\dot{a}}}^{2}=(\cdot)^{\mathrm{T}} Q_{\hat{a}}^{-1}(\cdot)$ Note that $P(\breve{a}=a)$ equals the probability of correct integer ambiguity estimation. It describes the expected success rate of GNSS ambiguity resolution.

\subsection{The Baseline Solution}

We are now in the position to determine the pdf of the 'float' baseline estimator Eq.(4). In order to determine this pdf, one needs to propagate the uncertainty of the 'float' solution, $\hat{a}$ and $\hat{b}$, as well as the uncertainty of the integer solution $\breve{a}$ through Eq.(4). Should one neglect the random character of the integer solution and therefore consider the ambiguity vector $\breve{a}$ as deterministic and equal to, say, $z$, then the pdf of $\breve{b}$ would equal the conditional baseline distribution

$$
p_{\hat{b} \hat{a}}(x \mid z)=\frac{1}{\sqrt{\operatorname{det} Q_{\hat{b} \hat{a}}}(2 \pi)^{\frac{1}{2} p}} \exp \left\{-\frac{1}{2}\|x-b(z)\|_{Q_{b \hat{b}}}^{2}\right\}(9)
$$

with conditional mean $b(z)=b-Q_{\hat{b} \hat{a}} Q_{\hat{a}}^{-1}(a-z)$, conditional variance matrix $Q_{\hat{b} \mid \hat{a}}=Q_{\hat{b}}-Q_{\hat{b} \hat{a}} Q_{\hat{a}}^{-1} Q_{\hat{a} \hat{b}}$ and $\|\cdot\|_{Q_{b \mid \hat{a}}}^{2}=(\cdot)^{\mathrm{T}} Q_{\hat{b} \mid \hat{a}}^{-1}(\cdot)$. However, since $\breve{a}$ is random and not deterministic, the conditional baseline distribution will give a too optimistic description of the quality of the 'fixed' baseline. To get a correct description of the 'fixed' baseline's pdf, the integer ambiguity's pmf needs to be considered. As the following theorem shows this results in a baseline distribution, which generally will be multi-modal.

Theorem 1 (Pdf of the 'float' baseline) Let the 'float' solution, $\hat{a}$ and $\hat{b}$, be normally distributed with mean $a \in Z^{n}$ and mean $b \in R^{p}$, and vc-matrix Eq.(2), let $\breve{a}$ be an admissible integer estimator and let the 'fixed' baseline $\breve{b}$ be given as in Eq.(4). The pdf of $\breve{b}$ reads then

$$
p_{\breve{b}}(x)=\sum_{z \in Z^{n}} p_{\hat{b} \mid \hat{a}}(x \mid z) P(\breve{a}=z)
$$

Note that, although the model Eq.(1) is linear and the observables normally distributed, the distribution of the 'fixed' baseline is not normal, but multi-modal (see
Fig.4). As the theorem shows, the 'fixed' baseline distribution equals an infinite sum of weighted conditional baseline distributions. These conditional baseline distributions $p_{\hat{b} \mid \hat{a}}(x \mid z)$ are shifted versions of one another. The size and direction of the shift is governed by $Q_{\hat{b} \hat{a}} Q_{\hat{a}}^{-1} z, z \in Z^{n}$. Each of the conditional baseline distributions in the infinite sum is down-weighted. These weights are given by the probability masses of the distribution of the integer ambiguity estimator $\breve{a}$. This shows that the dependence of the 'fixed' baseline distribution on the choice of integer estimator is only felt through the weights $P(\bar{a}=z)$.

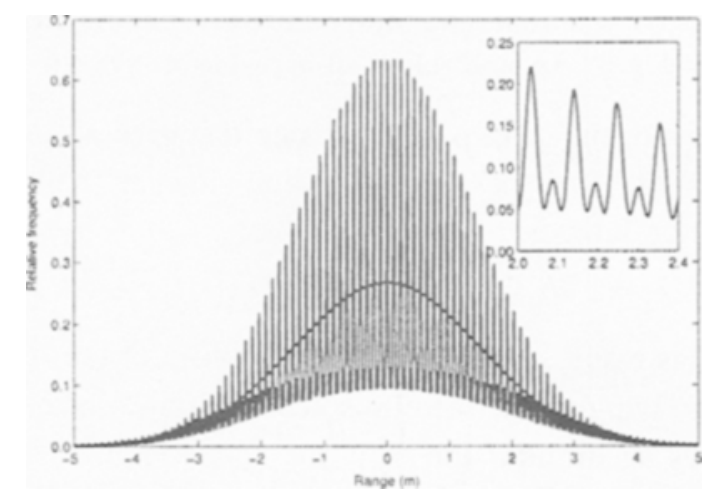

Fig.4 An example of the multi-modal pdf of $\breve{b}$ and corresponding uni-modal pdf of $\hat{b}$

\subsection{On the Quality of the 'Fixed' Baseline}

In order to describe the quality of the 'fixed' baseline, one would like to know how close one can expect the baseline estimate $\breve{b}$ to be to the unknown, but true baseline value $b$. As a measure of confidence, we take

$$
P(\breve{b} \in R)=\int_{R} p_{\breve{b}}(x) \mathrm{d} x, \text { with } R \subset R^{p}
$$

But in order to evaluate this integral, we first need to make a choice about the shape and location of the subset $R$. Since it is common practice in GNSS positioning to use the vc-matrix of the conditional baseline estimator as a measure of precision for the 'fixed' baseline, the vc-matrix $Q_{\hat{b} \mid \hat{a}}$ will be used to define the shape of the confidence region. For its location, we choose the confidence region to be centered at $b$. After all, we would like to know by how much the baseline estimate $\breve{b}$ can be expected to differ from the true, but unknown baseline value $b$. That is, one would like Eq.(11) to be a measure of the baseline's probability 
of concentration about $b$.

With these choices on shape and location, the region $R$ takes the form

$$
R=\left\{x \in R^{p} \mid(x-b)^{\mathrm{T}} Q_{\hat{b} \hat{a} \hat{a}}^{-1}(x-b) \leqslant \beta^{2}\right\}
$$

The size of the region can be varied by varying $\beta$. The following theorem shows how the baseline's probability of concentration Eq.(11) can be evaluated as a weighted sum of probabilities of noncentral Chi-square distributions.

Theorem 2 (The 'fixed' baseline's probability of concentration) Let $b$ be the 'fixed' baseline estimator, let $R$ be defined as in Eq.(12), and let $\chi^{2}\left(p, \lambda_{z}\right)$ denote the noncentral Chi-square distribution with $p$ degrees of freedom and non-centrality parameter $\lambda_{z}$. Then

$$
P(\breve{b} \in R)=\sum_{z \in Z^{n}} P\left(\chi^{2}\left(p, \lambda_{z}\right) \leqslant \beta^{2}\right) P(\breve{a}=z)
$$

with

$$
\lambda_{z}=\left\|\nabla \breve{b}_{z}\right\|_{Q_{b \hat{b}}}^{2} \text { and } \nabla \breve{b}_{z}=Q_{\hat{b} \hat{a}} Q_{\hat{a}}^{-1}(z-a)
$$

This result shows that the probability of the 'fixed' baseline lying inside the ellipsoidal region $R$ centered at $b$ equals an infinite sum of probability products. If one considers the two probabilities of these products separately, two effects are observed. First the probabilistic effect of shifting the conditional baseline estimator away from $b$ and secondly the probabilistic effect of the peakedness or non-peakedness of the ambiguity pmf. The second effect is related to the expected performance of ambiguity resolution, while the first effect has to do with the sensitivity of the baseline for changes in the values of the integer ambiguities. This effect is measured by the non-centrality parameter $\lambda_{z}$. Since the tail of a noncentral Chi-square distribution becomes heavier when the noncentrality parameter increases, while the degrees of freedom remain fixed, $P\left(\chi^{2}\left(p, \lambda_{z}\right) \leqslant \beta^{2}\right)$ gets smaller when $\lambda_{z}$ gets larger.

The two probabilities in the product reach their maximum values when $z=a$. The following corollary shows how these two maxima can be used to lower bound and to upper bound the probability $P(\breve{b} \in R)$. Such bounds are of importance for practical purposes, since it is difficult in general to evaluate Eq.(13) exactly. Corollary 1 (Lower and upper bounds) Let $\vec{b}$ be the 'fixed' baseline estimator and let $R$ be defined as in Eq.(12). Then

$$
P\left(\hat{b}_{\hat{a}=a} \in R\right) P(\breve{a}=a) \leqslant P(\breve{b} \in R) \leqslant P\left(\hat{b}_{\hat{k}=a} \in R\right)
$$

with

$$
P\left(\hat{b}_{\mid \hat{a}=a} \in R\right)=P\left(\chi^{2}(p, 0) \leqslant \beta^{2}\right.
$$

Note that the two bounds relate the probability of the 'fixed' baseline estimator to that of the conditional estimator and the ambiguity success rate. The above bounds become tight when the ambiguity success rate approaches one. This shows, although the probability of the conditional estimator always overestimates the probability of the 'fixed' baseline estimator, that the two probabilities are close for large values of the success rate. This implies that in case of GNSS ambiguity resolution, one should first evaluate the success rate $P(\breve{a}=a)$ and make sure that its value is close enough to one, before making any inferences on the basis of the distribution of the conditional baseline estimator. In other words, the (uni-modal) distribution of the conditional estimator is a good approximation to the (multi-modal) distribution of the bootstrapped baseline estimator, when the success rate is sufficiently close to one.

\section{Integer Bootstrapping}

\subsection{The Bootstrapped Estimator}

The distributional results presented so far hold for any admissible ambiguity estimator. The simplest way to obtain an integer vector from the real-valued 'float' solution is to round each of the entries of $\hat{a}$ to its nearest integer. The corresponding integer estimator reads therefore

$$
\breve{a}_{R}=\left(\left[\hat{a}_{1}\right] \cdots,\left[\hat{a}_{n}\right]\right)^{\mathrm{T}}
$$

where '[.]' denotes rounding to the nearest integer. The pullin region of this integer estimator equals the multivariate version of the unit square (see Fig.3).

Another relatively simple integer ambiguity estimator is the bootstrapped estimator. The bootstrapped estimator can be seen as a generalization of the previous estimator, Ref.[13] and Ref.[14]. It still makes use of integer rounding, but it also takes some of the correlation between the ambiguities into account. The bootstrapped estimator follows from a sequential conditional least-squares adjustment and it is computed as follows. If $n$ ambiguities are available, one starts with the first ambiguity $\hat{a}_{1}$, and rounds its value to the nearest integer. Having obtained the integer value of this first ambiguity, the real-valued estimates of all remaining ambiguities are then corrected by virtue of their correlation with the first ambiguity. Then the second, but now corrected, real-valued ambiguity estimate is 
rounded to its nearest integer. Having obtained the integer value of the second ambiguity, the real-valued estimates of all remaining $n-2$ ambiguities are then again corrected, but now by virtue of their correlation with the second ambiguity. This process is continued until all ambiguities are considered. We thus have the following definition.

Definition 2 (Integer bootstrapping) Let $\hat{a}=\left(\hat{a}_{1}, \cdots, \hat{a}_{n}\right)^{\mathrm{T}}$ $\in Z^{n}$ be the ambiguity 'float' solution and let $\breve{a}_{B}=\left(\breve{a}_{B, 1}, \cdots, \breve{a}_{B, n}\right)^{\mathrm{T}} \in Z^{n}$ denote the corresponding integer bootstrapped solution. The entries of the bootstrapped ambiguity estimator are then defined as

$$
\begin{aligned}
& \breve{a}_{B, 1}=\left[\hat{a}_{1}\right] \\
& \breve{a}_{B, 2}=\left[\hat{a}_{2 ! 1}\right]=\left[\hat{a}_{2}-\sigma_{21} \sigma_{1}^{-2}\left(\hat{a}_{1} \breve{a}_{B, 1}\right)\right] \\
& \vdots \\
& \breve{a}_{B, n}=\left[\hat{a}_{n \mid N}\right]=\left[\hat{a}_{n}-\sum_{j=1}^{n-1} \sigma_{n, j \mid J} \sigma_{j \mid J}^{-2}\left(\hat{a}_{j \mid J}-\breve{a}_{B, j}\right)\right]
\end{aligned}
$$

where '[ $]$ ' denotes the operation of rounding to the nearest integer, and $\sigma_{i, j l l}$ denotes the covariance between $\hat{a}_{i}$ and $\hat{a}_{j \mid J}$, and $\sigma_{j \mid J}$ is the variance of $\hat{a}_{j \mid J}$. The shorthand notation $\hat{a}_{i \mid l}$ stands for the $i$ th least-squares ambiguity obtained through a conditioning on the previous $I=\{1, \cdots,(i-1)\}$ g sequentially rounded ambiguities.

Note that the bootstrapped estimator is not unique. Changing the order in which the ambiguities appear in vector $\hat{a}$ will already produce a different bootstrapped estimator. Although the principle of bootstrapping remains the same, every choice of ambiguity parameterization has its own bootstrapped estimator.

\subsection{The Bootstrapped Pull-in Regions}

The pull-in regions for rounding are unit cubes centered at integer grid points. For bootstrap ping the shape of the pull-in regions will depend on the vc-matrix of the ambiguities. They will coincide with the unit cubes only in case the vc-matrix is a diagonal matrix. Bootstrapping reduces namely to rounding in the absence of any correlation between the ambiguities. The following theorem gives a description of the bootstrapped pull-in regions in the general case.

Theorem 3 (Bootstrapped pull-in regions) The pull-in regions of the bootstrapped ambiguity estimator $\bar{a}_{B}=$ $\left(\breve{a}_{B, 1} \cdots \breve{a}_{B, n}\right)^{\mathrm{T}} \in Z^{n}$ are given as

$$
S_{B, z}=\left\{x \in R^{n} \| c_{i}^{\mathrm{T}} L^{-1}(x-z) \mid \leqslant \frac{1}{2},\right.
$$

$$
i=1, \cdots, n\}, \quad \forall z \in Z^{n}
$$

where $L$ denotes the unique unit lower triangular matrix of the ambiguity vc-matrix decomposition $Q_{\hat{a}}=L D L^{\mathrm{T}}$ and $c_{i}$ denotes the $i$ th canonical unit vector having a 1 as its $i$ th entry and zeros otherwise.

That the bootstrapped estimator is indeed admissible, can now be seen as follows. The first two conditions of Definition 1 are easily verified using the definition of the bootstrapped estimator. Since every real-valued vector $\hat{a}$ will be mapped by the bootstrapped estimator to an integer vector, the pull-in regions $S_{B, z}$ cover $R^{n}$ without any gaps. There is also no

overlap between the pull-in regions, since - apart from boundary ties-any real-valued vector $\hat{a}$ is mapped to not more than one integer vector. To verify the last condition of Definition 1, we make use of Eq.(17). From

$$
\begin{aligned}
S_{B, z}= & \left\{x \in R^{n} \| c_{i}^{\mathrm{T}} L^{-1}(x-z) \mid \leqslant \frac{1}{2},\right. \\
i=1, \cdots, n\} & \\
= & \left\{x \in R^{n} \| c_{i}^{\mathrm{T}} L^{-1} y \mid \leqslant \frac{1}{2},\right. \\
x=y+z, i=1, \cdots n\} & \\
= & \left\{S_{B, 0}+z\right\}
\end{aligned}
$$

it follows that all bootstrapped pull-in regions are translated copies of $S_{B, 0}$. All pull-in regions have therefore the same shape and the same volume. Their volumes all equal 1 . This can be shown by transforming $S_{B, 0}$ to the unit cube centered at the origin. Consider the linear transformation $y=L^{-1} x$. Then

$$
L^{-1}\left(S_{B, 0}\right)=\left\{y \in R^{n} \| c_{\mathrm{i}}^{\mathrm{T}} y \mid \leqslant \frac{1}{2}, i=1, \cdots, n\right\}
$$

equals the unit cube centered at the origin. Since the determinant of the unit lower triangular matrix $L^{-1}$ equals one and since the volume of the unit cube equals one, it follows that the volume of $S_{B, 0}$ must equal one as well. To infer the shape of the bootstrapped pull-in region, we consider the two dimensional case first. Let the lower triangular matrix $L$ be given as

$$
L=\left[\begin{array}{ll}
1 & 0 \\
l & 1
\end{array}\right]
$$

Then

$$
\begin{aligned}
S_{B, 0} & =\left\{x \in R^{2} \| c_{i}^{T} L^{-1} x \mid \leqslant \frac{1}{2}, i=1,2\right\} \\
& =\left\{x \in R^{2} \| x_{1}\left|\leqslant \frac{1}{2},\right| x_{2}-l x_{1} \mid \leqslant \frac{1}{2}\right\}
\end{aligned}
$$

which shows that the two-dimensional pull-in region 
equals a parallelogram (see Fig.5). Its region is bounded by the two vertical lines $x_{1}=1 / 2$ and $x_{1}=-1 / 2$, and the two parallel slopes $x_{2}=l x_{1}+1 / 2$ and $x_{2}=l x_{1}-1 / 2$. The direction of the slope is governed by $l=\sigma_{21} \sigma_{1}^{-2}$. Hence, in the absence of correlation between the two ambiguities, the parallelogram reduces to the unit square. In higher dimensions the above construction of the pull-in region can be continued. In three dimensions for instance, the intersection of the pull-in region with the $x_{1} x_{2}$-plane remains a parallelogram, while along the third axis the pull-in region becomes bounded by two parallel planes.

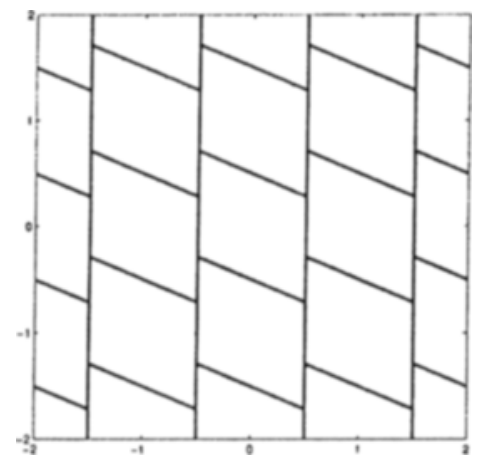

Fig.5 The two-dimensional pull-in regions of integer bootstrapping

\subsection{The Bootstrapped pmf}

Since the integer bootstrapped estimator is defined as $\breve{a}_{B}=z \Leftrightarrow \hat{a} \in S_{B}, z$, it follows that $P\left(\breve{a}_{B}=z\right)=$ $P\left(\hat{a} \in S_{B, z}\right)$. The pmf of $\breve{a}_{B}$ follows therefore as

$$
P\left(\breve{a}_{B}=z\right)=\int_{S_{B, z}} P_{\hat{a}}(x) \mathrm{d} x, z \in Z^{n}
$$

Hence, the probability that $\breve{a}_{B}$ coincides with $z$ is given by the integral of the pdf $p_{\hat{a}}(x)$ over the bootstrapped pull-in region $S_{B, z} \subset R^{n}$. The above expression holds for any distribution the 'float' ambiguities $\hat{a}$ might have. In most GNSS applications however, one usually assumes the vector of observables $y$ to be normally distributed. For that case the following theorem gives an exact expression of the bootstrapped pmf (see Fig.6).

Theorem 4 (The integer bootstrapped pmf) Let $\hat{a}$ be distributed as $N\left(a, Q_{\hat{a}}\right), a \in Z^{n}$, and let $\breve{a}_{B}$ be the corresponding integer bootstrapped estimator. Then

$$
\begin{aligned}
P\left(\vec{a}_{B}=z\right)= & \prod_{i=1}^{n}\left[\Phi\left(\frac{1-2 l_{i}^{\mathrm{T}}(a-z)}{2 \sigma_{\hat{a}_{i U}}}\right)\right. \\
& \left.+\Phi\left(\frac{1+2 l_{i}^{\mathrm{T}}(a-z)}{2 \sigma_{\hat{a}_{M}}}\right)-1\right], z \in Z^{n}
\end{aligned}
$$
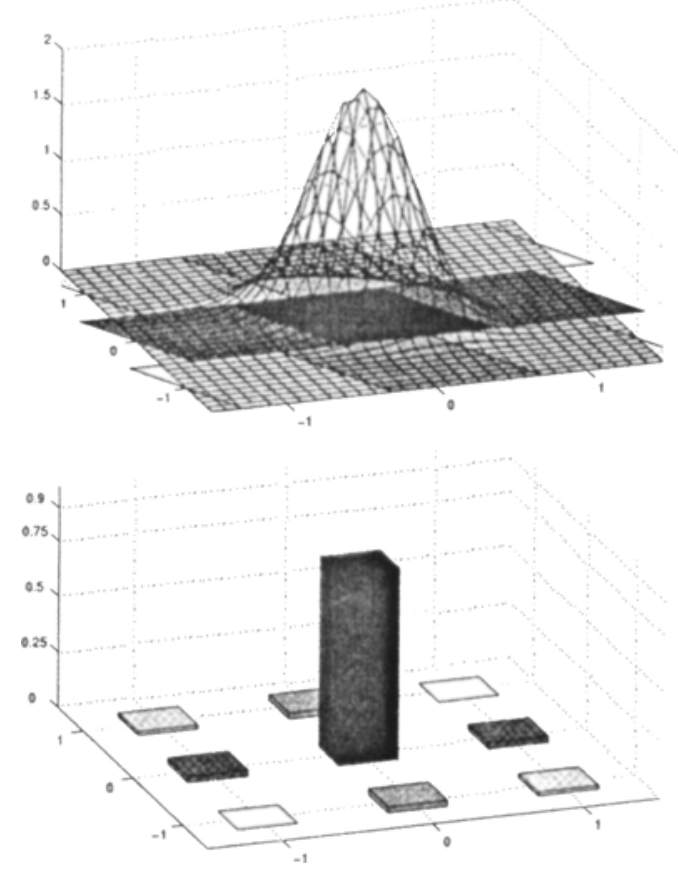

Fig.6 (top) The two-dimensional pdf of the 'float' solution $\hat{a}$ situated over the bootstrapped pull-in regions; (bottom) the two-dimensional pmf of the integer bootstrapped solution $\breve{a}_{B}$

with

$$
\Phi(x)=\int_{-\infty}^{x} \frac{1}{\sqrt{2 \pi}} \exp \left\{-\frac{1}{2} v^{2}\right\} \mathrm{d} v
$$

and with $l_{i}$ the $i$ th column vector of the unit lower triangular matrix $L^{-\mathrm{T}}$ and $\sigma_{\hat{a}_{i \mid l}}^{3}$ the variance of the $i$ th least-squares ambiguity obtained through a conditioning on the previous $I=\{1, \cdots,(i-1)\}$ ambiguities.

The bootstrapped pmf equals a product of univariate pmf's and is therefore easy to compute. Note that the bootstrapped pmf is completely governed by the ambiguity vc-matrix $Q_{\hat{a}}$. The pmf follows once the triangular factor $L$ and the diagonal matrix $D$ of the decomposition $Q_{\hat{a}}=L D L^{\mathrm{T}}$ are given. The above result also shows that the bootstrapped pmf is symmetric about the mean of $\hat{a}$. This implies that the bootstrapped estimator $\vec{a}_{B}$ is an unbiased estimator of $a \in Z^{n}$. Since the 'float' solutions, $\hat{a}$ and $\hat{b}$, are unbiased too, it follows from taking the expectation of Eq.(4) that the bootstrapped baseline is also unbiased.

For the purpose of predicting the success of ambiguity resolution, the probability of correct integer estimation is of particular interest. For the bootstrapped estimator this success rate is given in the following corollary.

Corollary 2 (The bootstrapped success rate) The boots- 
trapped probability of correct integer estimation (the success rate) is given as

$$
P\left(\breve{a}_{B}=a\right)=\prod_{i=1}^{n}\left[2 \Phi\left(\frac{1}{2 \sigma_{\hat{a}_{\mid l}}}\right)-1\right]
$$

The method of integer bootstrapping is easy to implement and it does not need, as opposed to the method of integer leastsquares (see next section), an integer search for computing the sought for integer solution. However, as it was mentioned earlier, the outcome of bootstrapping depends on the chosen ambiguity parameterization. Bootstrapping of DD ambiguities, for instance, will produce an integer solution which generally differs from the integer solution obtained from bootstrapping of reparameterized ambiguities. Since this dependency also holds true for the bootstrapped pmf, one still has some important degrees of freedom left for improving Eq.(20).

In order to improve the bootstrapped success rate, one should work with decorrelated ambiguities instead of with the original ambiguities. The method of bootstrapping performs relatively poor, for instance, when applied to the DD ambiguities. This is due to the usually high correlation between the DD ambiguities. Bootstrapping should therefore be used in combination with the decorrelating Z-transformation of the LAMBDA method, see Ref.[15],[16]. This transformation decorrelates the ambiguities further than the best reordering would achieve and thereby reduces the values of the sequential conditional variances. By reducing the values of the sequential conditional variances, the bootstrapped success rate gets enlarged.

It may however happen that it is simply not possible to resolve the complete vector of ambiguities with sufficient probability. As an alternative of resolving the complete vector of ambiguities, one might then consider resolving only a subset of the ambiguities. The idea of partial ambiguity resolution is based on the fact that the success rate will generally increase when fewer integer constraints are imposed. However, in order to apply partial ambiguity resolution, one first will have to determine which subset of ambiguities to choose. It will be clear that this decision should be based on the precision of the 'float' ambiguities. The more precise the ambiguities, the larger the ambiguity success rate. It is at this point where the decorrelation step of the LAMBDA method and the bootstrapping principle can be applied. Once the transformed and decorrelated ambiguity vc-matrix is obtained, the construction of the subset proceeds in a sequential fashion. One first starts with the most precise ambiguity, say $\hat{z}_{1}$, and computes its success rate $P\left(\breve{a}_{1}=z_{1}\right)$. If this success rate is large enough, one continues and determines the most precise pair of ambiguities, say $\left(\hat{z}_{1}, \hat{z}_{2}\right)$. If their success rate is still large enough, one continues again by trying to extend the set. This procedure continues until one reaches a point where the corresponding success rate becomes unacceptably small. When this point is reached, one can expect that the previously identified ambiguities can be resolved successfully.

Once the subset for partial ambiguity resolution has been identified, one still needs to determine what this will do to improve the baseline estimator. After all, being able to successfully resolve the ambiguities does not necessarily mean that the 'fixed' solution is significantly better than the 'float' solution. The theory presented in the previous sections provides the necessary tools for performing such an evaluation.

\section{Integer Least-Squares}

\subsection{The ILS Estimator}

When using the least-squares principle, the GNSS model can be solved by means of the minimization problem

$$
\min _{a, b}\|y-A a-B b\|_{Q_{y}}^{2}, a \in Z^{n}, b \in R^{p}
$$

with $Q_{y}$ the vc-matrix of the GNSS observables. This type of least-squares problem was first introduced in Ref.[15] and has been coined with the term 'integer least-squares'. It is a nonstandard least-squares problem due to the integer constraints $a \in Z^{n}$, see Ref.[16],[17]. The solution of Eq.(21) is consistent with the three solution steps of section 1 . This can be seen as follows. It follows from the orthogonal decomposition

$$
\begin{aligned}
\|y-A a-B b\|_{Q_{y}}^{2}= & \|\hat{e}\|_{Q_{y}}^{2}+\|\hat{a}-a\|_{Q_{\dot{a}}}^{2} \\
& +\|\hat{b}(a)-b\|_{Q_{\hat{b} \hat{\bar{b}}}^{2}}^{2}
\end{aligned}
$$

with $\hat{e}=y-A \hat{a}-B \hat{b}$ and $\hat{b}(a)=\hat{b}-Q_{\hat{b} \hat{a}} Q_{\hat{a}}^{-1}(\hat{a}-a)$, that the sought for minimum is obtained when the second term on the right-hand side is minimized for $a \in Z^{n}$ and the last term is set to zero. The integer least-squares (ILS) estimator of the ambiguities is therefore defined as follows.

Definition 3 (Integer least-squares) Let $\hat{a}=\left(\hat{a}_{1}, \cdots, \hat{a}_{n}\right)^{\mathrm{T}}$ $\in R^{\prime \prime}$ be the ambiguity 'float' solution and let 
$\breve{a}_{\mathrm{LS}} \in Z^{n}$ denote the corresponding integer leastsquares solution. Then

$$
\breve{a}_{\mathrm{LS}}=\arg \min _{z \in Z^{n}}\|\hat{a}-z\|_{Q_{a}}^{2}
$$

In contrast to integer rounding and integer bootstrapping, an integer search is needed to compute $\breve{a}_{\mathrm{LS}}$. Although we will refrain from discussing the computational intricacies of ILS estimation, the conceptual steps of the computational procedure will be described briefly. The ILS procedure is mechanized in the GNSS LAMBDA (Least-squares AMBiguity Decorrelation Adjustment) method, which is currently one of the most applied methods for GNSS carrier phase ambiguity resolution. For more information on the LAMBDA method, we refer to e.g. Ref.[15,16,18] or to the textbooks Ref.[6,10,11]. Practical results obtained with it can be found, for example, in Ref.[19-28].

The main steps as implemented in the LAMBDA method are as follows. One starts by defining the ambiguity search space

$$
\Omega_{a}=\left\{a \in Z^{n} \mid(\hat{a}-a)^{\mathrm{T}} Q_{\hat{a}}^{-1}(\hat{a}-a) \leqslant \chi^{2}\right\}
$$

with $\chi^{2} a$ to be chosen positive constant. The boundary of this search space is ellipsoidal. It is centered at $\hat{a}$, its shape is governed by the vc-matrix $Q_{\hat{a}}$ and its size is determined by $\chi^{2}$. In case of GNSS, the search space is usually extremely elongated, due to the high correlations between the ambiguities. Since this extreme elongation usually hinders the computational efficiency of the search, the search space is first transformed to a more spherical shape,

$$
\Omega_{z}=\left\{z \in Z^{n} \mid(\hat{z}-z)^{\mathrm{T}} Q_{\hat{z}}^{-1}(\hat{z}-z) \leqslant \chi^{2}\right\}
$$

using the admissible ambiguity transformations $\hat{z}=Z^{\mathrm{T}} \hat{a}$, $Q_{\hat{\hat{\imath}}}=Z^{\mathrm{T}} Q_{\hat{a}} Z$. Ambiguity transformations $Z$ are said to be admissible when both $Z$ and its inverse $Z^{-1}$ have integer entries. Such matrices preserve the integer nature of the ambiguities. In order for the transformed search space to become more spherical, the volume-preserving $Z$-transformation is constructed as a transformation that decorrelates the ambiguities as much as possible. Using the triangular decomposition of $Q_{\tilde{\Sigma}}$, the left-hand side of the quadratic inequality in Eq.(25) is then written as a sum-of-squares:

$$
\sum_{i=1}^{n} \frac{\left(\hat{z}_{i I I}-z_{i}\right)^{2}}{\sigma_{i I I}^{2}} \leqslant \chi^{2}
$$

On the left-hand side one recognizes the conditional leastsquares estimator $\hat{z}_{\text {ill }}$, which follows when the conditioning takes place on the integers $z_{1}, z_{2}, \ldots, z_{i-1}$. Using the sum-ofsquares structure, one can finally set up the $n$ intervals which are used for the search. These sequential intervals are given as

$$
\begin{aligned}
\left(\hat{z}_{1}-z_{1}\right)^{2} & \leqslant \sigma_{1}^{2} \chi^{2} \\
\left(\hat{z}_{211}-z_{2}\right)^{2} & \leqslant \sigma_{211}^{2}\left(\chi^{2}-\frac{\left(\hat{z}_{1}-z_{1}\right)^{2}}{\sigma_{1}^{2}}\right)
\end{aligned}
$$

In order for the search to be efficient, one not only would like the vc-matrix $Q_{\hat{乏}}$ to be as close as possible to a diagonal matrix, but also that the search space does not contain too many integer grid points. This requires the choice of a small value for $\chi^{2}$, but one that still guarantees that the search space contains at least one integer grid point. Since the bootstrapped estimator is so easy to compute and at the same time gives a good approximation to the ILS estimator (see section 3.4), the bootstrapped solution is an excellent candidate for setting the size of the ambiguity search space. Following the decorrelation step $\hat{z}=Z^{\mathrm{T}} \hat{a}$, the LAMBDA-method therefore uses, as one of its options, the bootstrapped solution $\breve{Z}_{\mathrm{B}}$ for setting the size of the ambiguity search space as

$$
\chi^{2}=\left(\hat{z}-\breve{z}_{B}\right)^{\mathrm{T}} Q_{\hat{z}}^{-1}\left(\hat{z}-\breve{z}_{B}\right)
$$

In this way one can work with a very small search space and still guarantee that the sought for integer least-squares solution is contained in it.

\subsection{The ILS Pull-in Region}

The pull-in regions of integer rounding are unit cubes, while those of integer bootstrapping are multivariate versions of parallelograms. To determine the ILS pull-in regions we need to know the set of 'float' solutions $\hat{a} \in R^{n}$ that are mapped to the same integer vector $z \in Z^{n}$. This set is described by all $x \in R^{n}$ that satisfy $z=\arg \min _{u \in Z^{n}}\|x-u\|_{Q_{\dot{a}}}^{2}$. The ILS pull-in region that belongs to the integer vector $z$ follows therefore as

$$
S_{\mathrm{I} S, z}=\left\{x \in R^{n} \mid\|x-z\|_{Q \hat{a}}^{2} \leqslant\|x-u\|_{Q_{a}}^{2}, \forall u \in Z^{n}\right\}
$$

It consists of all those points which are closer to $z$ than to any other integer point in $R^{n}$. The matric used for measuring these distances is determined by the vc-matrix $Q_{\hat{a}}$. Based on Eq.(29), one can give a representation of the ILS pull-in regions that resembles the representation of the bootstrapped pull-in regions. This representation 
reads as follows.

Theorem 5 (ILS pull-in regions) The pull-in regions of the ILS ambiguity estimator $\breve{a}_{1 S} \in Z^{n}$ are given as

$$
\begin{aligned}
S_{\mathrm{IS}, z} & =\bigcap_{c_{i} \in Z^{n}}\left\{x \in R^{n} \| c_{i}^{\mathrm{T}} Q_{\hat{a}}^{-1}(x-z) \mid\right. \\
& \left.\leqslant \frac{1}{2}\left\|c_{i}\right\|_{Q_{\dot{a}}}^{2}\right\}, \forall z \in Z^{n}
\end{aligned}
$$

This shows that the ILS pull-in regions are constructed from intersecting half-spaces. One can also show that at most $2^{n}-1$ pairs of such half spaces are needed for constructing the pullin region. The ILS pull-in regions are convex, symmetric sets of volume 1 , which satisfy the conditions of Definition 1. The ILS estimator is therefore admissible. The ILS pull-in regions are hexagons in the two-dimensional case (see Fig.7).

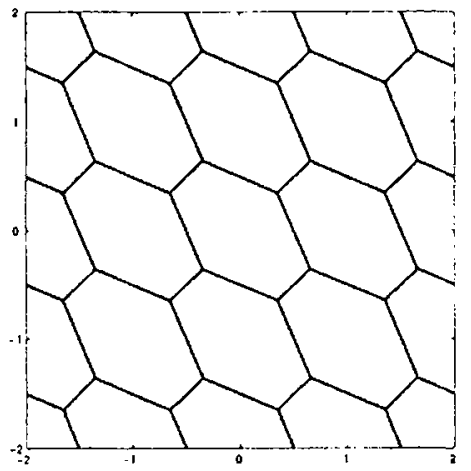

Fig.7 The two-dimensional ILS pull-in regions

\subsection{Maximizing the Success Rate}

Although various integer estimators exist which are admissible, some may be better than others. Having the problem of GNSS ambiguity resolution in mind, one is particularly interested in the estimator which maximizes the probability of correct integer estimation. This probability equals $P(\breve{a}=a)$, but it will differ for different ambiguity estimators. The following theorem, due to Ref.[29], shows that the ILS estimator maximizes the probability of correct integer estimation.

Theorem 6 (ILS is optimal) Let the pdf of the 'float' solution $\hat{a}$ be given as

$$
p_{a}(x)=\sqrt{\operatorname{det}\left(Q_{\hat{a}}^{-1}\right)} G\left(\|x-a\|_{Q_{\dot{a}}}^{2}\right)
$$

where $G: R \mapsto[0, \infty)$ is decreasing and $Q_{\hat{a}}$ is positivedefinite. Then

$$
P\left(\bar{a}_{\mathrm{LS}}=a\right) \geqslant P(\bar{a}=a)
$$

for any admissible estimator $\breve{a}$.

This theorem gives a probabilistic justification for using the ILS estimator. For GNSS ambiguity resolution it shows, that one is better off using the ILS estimator than any other admissible integer estimator. The family of distributions defined in Eq.(31), is known as the family of elliptically contoured distributions. Several important distributions belong to this family. The multivariate normal distribution can be shown to be a member of this family by choosing $G(x)=(2 \pi)^{-\frac{n}{2}} \exp \left(-\frac{1}{2} x\right), x \in R$. Another member is the multivariate $t$-distribution.

As a direct consequence of the above theorem we have the following corollary.

Corollary 3 (The effect of the weight matrix) Let $\Sigma$ be any positive-definite matrix of order $n$ and define

$$
\breve{a}_{\Sigma}=\arg \min _{z \in Z^{n}}\|\hat{a}-z\|_{\Sigma}^{2}
$$

Then $\breve{a}_{\Sigma}$ is admissible and

$$
P\left(\breve{a}_{\mathrm{LS}}=a\right) \geqslant P\left(\breve{a}_{\Sigma}=a\right)
$$

In order to prove the corollary, we only need to show that $\breve{a}_{\Sigma}$ is admissible. Once this has been established, the stated result Eq.(34) follows from theorem 6 . The admissibility can be shown as follows. The first two conditions of Definition 1 are satisfied, since the ILS-map produces - apart from boundary ties a unique integer vector for any 'float' solution $\hat{a} \in R^{n}$. And since $\breve{a}_{\Sigma}=\arg \min _{z \in Z^{n}}\|\hat{a}-u-z\|_{\Sigma}^{2}+u$ holds true for any integer $u \in Z^{n}$, also the integer remove-restore technique applies.

As the corollary shows, a proper choice of the data weight matrix is also of importance for ambiguity resolution. The choice of weights is optimal when the weight matrix equals the inverse of the ambiguity vc-matrix. A too optimistic precision description or a too pessimistic precision description, will both result in a less than optimal ambiguity success rate. In the case of GNSS, the observation equations (the functional model) are sufficiently known and well documented. However, the same can not yet be said of the vc-matrix of the GNSS data. In the many GNSS textbooks available, we will usually find only a few comments, if any, on this vc-matrix. Examples of studies that have been reported in the literature are: Ref.[30-33], who studied the elevation dependence of the observation variances; Ref.[25,34], who considered time correlation and cross correlation; and Ref.[35-37], who considered the inclusion of stochastic ionospheric constraints.

\subsection{Bounding the ILS Success Rate}

A very useful application of theorem 6 is that it shows how one can lower bound the ILS probability of correct integer estimation. This is particularly useful since the ILS success rate is usually difficult to compute.

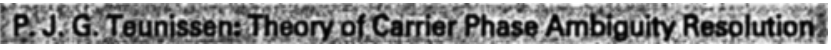


This is due to the rather complicated geometry of the ILS pull-in region. The bootstrapped success rate is a good candidate for the ILS success rates' lower bound. The bootstrapped success rate is easy to compute and it becomes a sharp lower bound when applied to the decorrelated ambiguities $\hat{z}=Z^{\mathrm{T}} \hat{a}$. In fact, at present, the bootstrapped success rate is the sharpest available lower bound of the ILS success rate.

Apart from having a lower bound, it is also useful to have an upper bound available. For obtaining an upper bound one can make use of the geometric mean of the ambiguity conditional variances. This geometric mean is referred to as the Ambiguity Dilution of Precision $(\mathrm{ADOP})$ and it is given as

$$
\mathrm{ADOP}=\sqrt{\operatorname{det} Q_{a}}{ }^{\frac{1}{n}} \text { (cycles) }
$$

Note that this scalar measure of the ambiguity precision is invariant for the admissible volume preserving ambiguity transformations. With the ADOP one can obtain an upper bound by making use of the fact that the probability content of the ILS pull-in region $S_{L S ; a}$ would be maximal if its shape would coincide with that of the ambiguity search space, while its volume would still be constrained to 1 . We have the following bounds for the ILS success rate.

Theorem 7 (Bounds on the IIS success rate) The ILS success rate $P\left(\breve{a}_{\mathrm{LS}}=a\right)$ is bounded from below and from above as

$$
P\left(\breve{z}_{\mathrm{B}}=z\right) \leqslant P\left(\breve{a}_{\mathrm{LS}}=a\right) \leqslant\left(\chi^{2}(n, 0) \leqslant \frac{c_{n}}{\mathrm{ADOP}^{2}}\right)
$$

with $\quad c_{n}=\left(\frac{n}{2} \Gamma\left(\frac{n}{2}\right)\right)^{2 / n} / \pi$

\section{The PDF of the Ambiguity Resi- duals}

Having determined the various probabilistic properties of the 'fixed' ambiguities and 'fixed' baseline solution, we are now in a position to draw our attention to the ambiguity residuals. In order to determine their pdf, we first need the the joint distribution of the 'float' ambiguities, $\hat{a}$, and the 'fixed' ambiguities, $\breve{a}$. The joint distribution of $\hat{a}$ and $\bar{a}$ will be denoted as $p_{\hat{a}, \bar{a}}(x, z)$. We have the following result.

Theorem 8 (The joint distribution of 'float' and 'fixed' ambiguities) Let $p_{\hat{a}}(x)$ be the pdf of $\hat{a}$ and let the integer ambiguity estimator be defined as $\breve{a}=\sum_{z \in Z^{n}} z s_{z}(\hat{a})$, with $s_{z}(x)$ the indicator function of the pull-in region $S_{z} \subset R^{n}, z \in Z^{n}$. The joint distribution of $\hat{a}$ and $\breve{a}$ is then given as

$$
p_{\hat{a}, \breve{a}}(x, z)=p_{\hat{a}}(x) s_{\mathfrak{z}}(x), x \in R^{n}, z \in Z^{n}
$$

For the proof we refer to Ref.[4].

The joint distribution Eq.(37) can be used to determine the distribution of functions of $\hat{a}$ and $\breve{a}$. An important example of such a function is the ambiguity residual. We define it as

$$
\breve{\varepsilon}=\hat{a}-\breve{a}
$$

We will determine the distribution of $\breve{\varepsilon}$ in two steps. We first determine the joint distribution of $\breve{\mathcal{E}}$ and $\breve{a}$ and then determine the marginal distribution $p_{\breve{\varepsilon}}(x)$ by means of summation. In deriving the joint distribution, we make use of the following transformation law for probability density functions. Let two random vectors $u$ and $v$ be related as $v=T u+t$, with $T$ and $t$ known, and matrix $T$ invertible. The pdf of $v$ can then be expressed in the pdf of $u$ as $p_{v}(v)=\left|\operatorname{det} T^{-1}\right| p_{u}\left(T^{-1}(v-t)\right)$.

By using the invertible transformation

$$
\left[\begin{array}{c}
\breve{\varepsilon} \\
\breve{a}
\end{array}\right]=\left[\begin{array}{cc}
I_{n} & -I_{n} \\
0 & I_{n}
\end{array}\right]\left[\begin{array}{l}
\hat{a} \\
\breve{a}
\end{array}\right]
$$

we can express the joint distribution of $\breve{\varepsilon}$ and $\breve{a}$ in the joint distribution of $\hat{a}$ and $\breve{a}$.This gives $p_{\widetilde{\varepsilon}, \bar{a}}(x, z)$. $=p_{\hat{a}, \bar{a}}(x+z, z)$. If we now make use of Eq.(37), we obtain $p_{\tilde{\varepsilon}, \tilde{a}}(x, z)=p_{\hat{a}}(x+z) s_{0}(x), x \in R^{n}, z \in Z^{n}$. The pdf of the ambiguity residuals follows then from summing this joint distribution over all integers,

$$
p_{\breve{\varepsilon}}(x)=\sum_{z \in Z^{n}} p_{\hat{a}}(x+z) s_{0}(x), x \in R^{n}, z \in Z^{n}
$$

In order to show how the distribution of the ambiguity residuals is constructed from the distribution of the 'float' ambiguities, a visualization of the steps involved is given in Fig.8 for the one-dimensional case $(n=1)$. This figure shows the four distributions, $p_{\hat{a}}(x)$ (top left), $p_{\hat{a}, \tilde{a}}(x, z)$ (top right), $p_{\bar{\varepsilon}, \bar{a}}(x, z)$ (bottom left) and $p_{\tilde{\varepsilon}}(x)$ (bottom right). For each integer $z \in Z^{n}$ the joint distribution $p_{\hat{a}, \bar{a}}(x, z)$ is composed of slices from the marginal distribution $p_{\hat{a}}(x)$ located at $(z, z)$. Translating these slices parallel to the $x$-axis to the line $x=0$ gives the joint $p_{\tilde{\varepsilon}, \bar{a}}(x, z)$. A further translation along the $z$-axis to the origin then finally provides $p_{\tilde{\varepsilon}}(x)$. The distribution of the ambiguity residuals is clearly non-Gaussian. We have $p_{\breve{\varepsilon}}(x)=0$ 
for all $x \notin S_{0}$. This implies that the norm of the vector of ambiguity residuals is always bounded irrespective the values taken by the ambiguity 'float' solution $\hat{a}$.For the one-dimensional case we have $\breve{\varepsilon}=[-1 / 2,+1 / 2]$. For the higher dimensional case the bound depends on the shape of the pull-in region and therefore on the type of integer estimator chosen. The fact that the ambiguity residuals are bounded has an important implication. It implies that the difference between the 'float' baseline solution $\hat{b}$ and the 'fixed' baseline solution $\breve{b}$ is also bounded, irrespective the values taken by the 'float' ambiguities (see Fig.9).
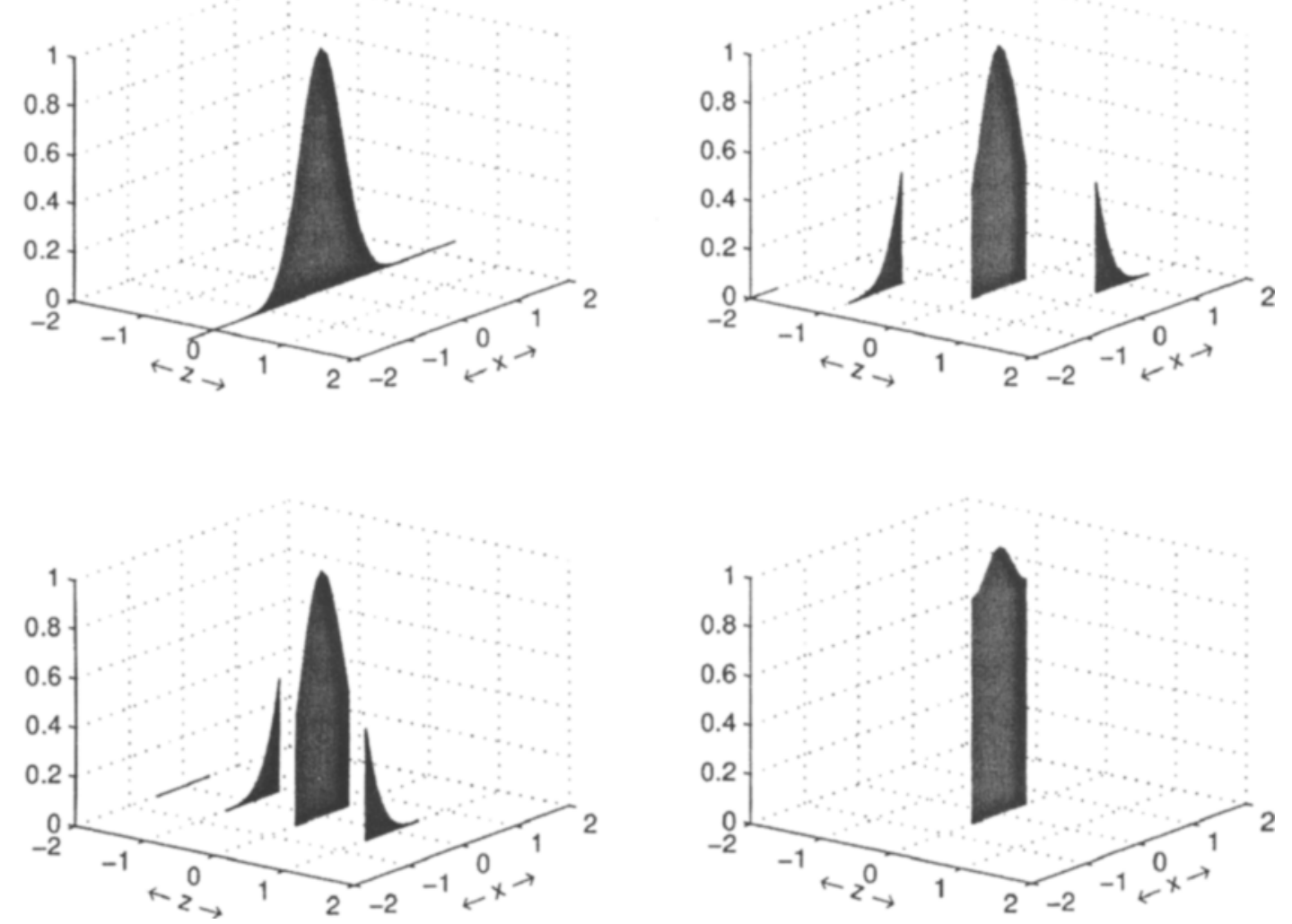

Fig.8 The construction of $p_{\breve{\varepsilon}}(x)$ from $p_{\hat{a}}(x)$.(top left)pdf $p_{\hat{a}}(x)$,(top right)joint pdf $p_{\hat{a}, \breve{a}}(x, z)$, (bottom left) joint pdf $p_{\breve{\varepsilon}, \breve{a}}(x, z)$, (bottom right) pdf $p_{\breve{\varepsilon}}(x)$

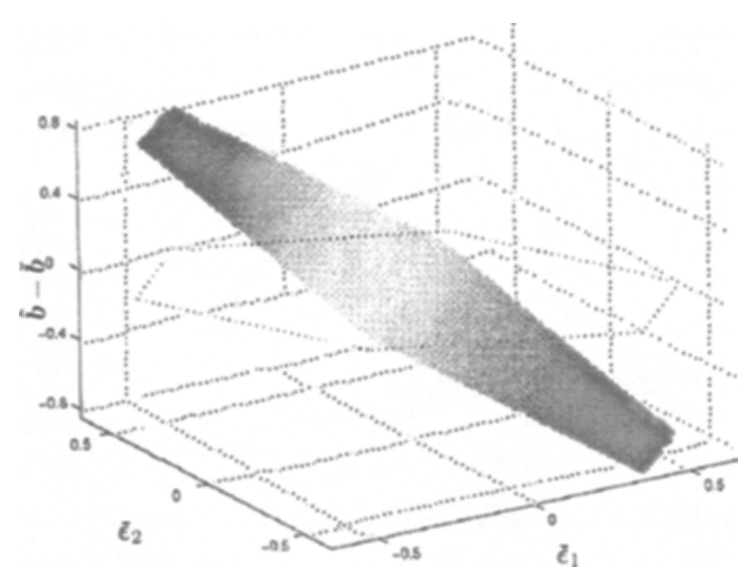

Fig.9 An example showing the bounded influence of the ambiguity residuals on the difference between the 'float' and 'fixed' baseline solution
The distribution $p_{\tilde{\varepsilon}}(x)$ can have different shapes. There are two extreme cases between which one can discriminate, namely the uniform distribution and the impulse function distribution. In order to understand these two extreme cases, consider what happens when the precision of the 'float' ambiguities, and thereby the peakedness of $p_{\hat{u}}(x)$, is varied. When the 'float' distribution $p_{\hat{a}}(x)$ gets more peaked, which happens when the 'float' ambiguities get more precise, the pdf $p_{\bar{\varepsilon}}(x)$ gets more peaked as well. However, since all the probability mass of $p_{\tilde{\varepsilon}}(x)$ is located within the pull-in region $S_{0}$, the peakedness of $p_{\tilde{\varepsilon}}(x)$ will only start to manifest itself when $p_{\hat{a}}(x)$ is sufficiently peaked in relation to the size of the pullin region. When this is not the case, the distribution of the ambiguity residuals will remain flat and therefore be close to $s_{0}(x)$, which is the 
uniform distribution for the pull-in region $S_{0}$. The 'float' distribution $p_{\hat{a}}(x)$ may be considered peaked in relation to the size of the pull-in region, when most of its probability mass is located within $S_{0}$.. This happens when the ambiguity success rate is sufficiently close to one, in which case the two distributions will also not differ by much. Further improvement of the precision of the ambiguities will then in the limit produce an impulse function for both $p_{\hat{a}}(x)$ and $p_{\tilde{\varepsilon}}(x)$ (see Fig. 10 for
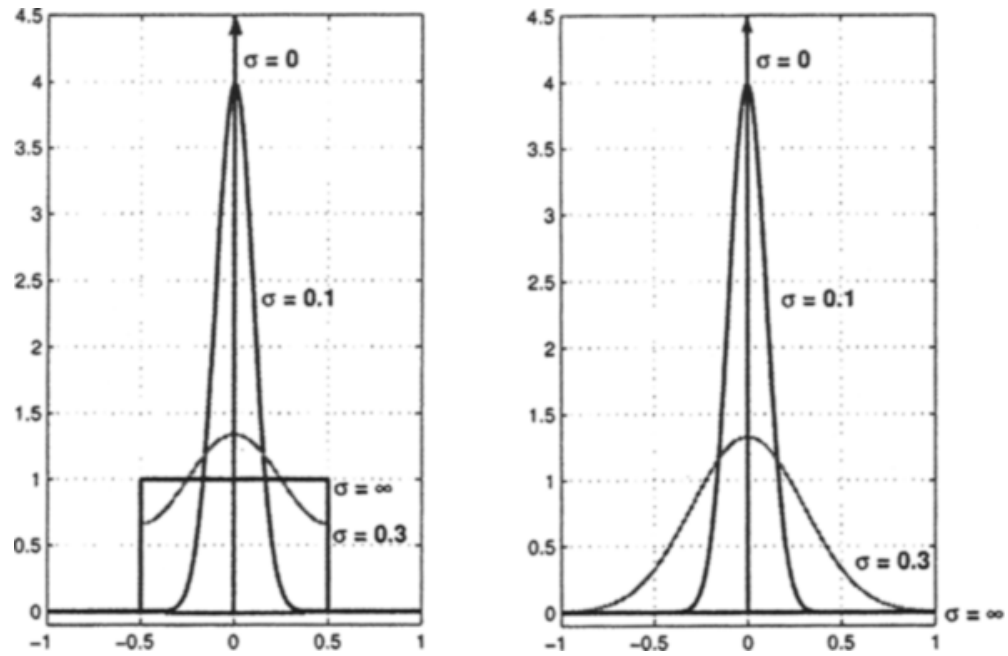

Fig.10 One dimensional pdf for different values of the ambiguity standard deviation (in cycles): (left) pdf of ambiguity residual, (right) pdf of corresponding 'float' ambiguity

the one-dimensional case). The distribution of the ambiguity residuals is symmetric and independent of the unknown integer ambiguity vector $a \in Z^{n}$. It gets its symmetry inherited from the 'float' distribution $p_{\hat{a}}(x)$, while the independence of $a \in Z^{n}$ follows from $\sum_{z \in Z^{n}} p_{\hat{a}}(x+a+z)=\sum_{z \in Z^{n}} p_{\hat{a}}(x+z)$. The point of symmetry of the distribution is the origin. This implies that the mean of the ambiguity residual equals zero,

$$
E\{\breve{\varepsilon}\}=0
$$

This result combined with the fact the pdf of $\breve{\mathcal{E}}$ is completely known once the precision of the 'float' ambiguities is given and once the choice of integer ambiguity estimator is made, allows one for the first time to formulate rigorous tests for the integerness of the parameters.

Acknowledgement: The author acknowledges the help of Sandra Verhagen and Peter Joosten in preparing (figures and formatting) the present contribution.

\section{References}

[1] Betti B, Crespi M, Sanso F. A Geometric Illustration of Ambiguity Resolution in GPS Theory and a Bayesian Approach. Manuscr Geod, 1993,18: 317-330.

[2] Gundlich B, Koch K-R. Confidence Regions for GPS
Baselines by Bayesian Statistics. Journal of Geodesy, 2002, 76: 55-62.

[3] Gundlich B, Teunissen P J G. Multiple Models: Fixed, Switching and Interacting. Proceedings V Hotine-Marussi Symposium, Matera, Italy, 2002.8.

[4] Teunissen P J G. The Parameter Distributions of the Integer GPS Model. Journal of Geodesy, 2002, 76: 41-48.

[5] Verhagen S, Teunissen P J G. PDF Evaluation of the Ambiguity Residuals. Proceedings $V$ Hotine-Marussi Symposium, Matera, Italy, 2002. 8.

[6] Hofmann-Wellenhof B, Lichtenegger H, Collins J. Global Positioning System: Theory and Practice. 5th Ed. New York: Springer Verlag, 2001.

[7] Leick A. GPS Satellite Surveying. 2nd Ed. New York: John Wiley, 1995.

[8] Misra P, Enge P. Global Positioning System: Signals, Measurements, and Performance. Massachusetts: GangaJamuna Press. 2001.

[9] Parkinson B, Spilker J J (Eds). GPS: Theory and Applications, Vols 1 and 2, Washington DC: AIAA,1996.

[10] Strang G, Borre K. Linear Algebra, Geodesy, and GPS. Wellesley: Wellesley-Cambridge Press, 1996.

[11] Teunissen P J G, Kleusberg A (Eds). GPS for Geodesy, 2nd Enlarged Ed. New York: Springer Verlag, 1998.

[12] Teunissen P J G. The Probability Distribution of the GPS Baseline for a Class of Integer Ambiguity Estimators. Joumal of Geodesy, 1999, 73: 275-284. 
[13] Teunissen P J G. Success Probability of Integer GPS Ambiguity Rounding and Bootstrapping. Journal of Geodesy, 1998, 72: 606-612.

[14] Teunissen P J G. The Probability Distribution of the Ambiguity Bootstrapped GNSS Baseline. Journal of Geodesy, 2001, 75: 267-275.

[15] Teunissen P J G. Least-Squares Estimation of the Integer GPS Ambiguitics. Invited Lecture, Section IV Theory and Methodology. IAG General Meeting, Beijing, China, August 1993.

[16] Teunissen P J G. The Least-Squares Ambiguity Decorrelation Adjustment: A Method for Fast GPS Integer Ambiguity Estimation. Journal of Geodesy, 1995, 70: 65-82.

[17] Hassibi A, Boyd S. Integer Parameter Estimation in Linear Models with Applications to GPS. IEEE Transactions on Signal Processing, 1998, 46(11): 2938-2952.

[18] de Jonge P J, Tiberius C C J M. The LAMBDA Method for Integer Ambiguity Estimation: Implementation Aspects. Delft: Publications of the Delft Computing Centre, LGR-Series 1996, 12.

[19] Boon F, Ambrosius B. Results of Real-Time Applications of the LAMBDA Method in GPS Based Aircraft Landings. Canada, Banff:Proceedings KIS97, 1997. 339-345.

[20] Boon F, de Jonge P J, Tiberius C C J M.Precise Aircraft Positioning by Fast Ambiguity Resolution Using Improved Troposphere Modelling. USA Kansas:Proceedings ION GPS-97, 1997, 2:1877-1884.

[21] Cox D B, Brading J D W. Integration of LAMBDA Ambiguity Resolution with Kalman Filter for Relative Navigation of Spacecraft. USA, San Diego:Proceedings ION NTM-99, 1999. 739-745.

[22] de Jonge P J, Tiberius C C J M. Integer Estimation with the LAMBDA Method. Boulder, Colorado: Proceedings IAG Symposium No. 115, 'GPS Trends in Terrestrial, Airborne and spaceborne applications', Beutler G Eds, New York: Springer Verlag, 1996, 280-284.

[23] de Jonge, $\mathrm{P}$ J, Tiberius $\mathrm{C} \mathrm{C} \mathrm{J} \mathrm{M.} \mathrm{Teunissen} \mathrm{P} \mathrm{J} \mathrm{G.}$ Computational Aspects of the LAMBDA Method for GPS Ambiguity Resolution. Proceedings ION GPS-96, USA, Missouri, Kansas, 1996. 935-944.

[24] Han S. Ambiguity Resolution Techniques Using Integer
Lcast-Squares Estimation for Rapid Static or Kinematic Positioning. Symposium Satellite Navigation Technology 1995, Brisbane, Australia, 1995.10.

[25] Jonkman N F. Integer GPS Ambiguity Estimation without the Receiver-Satellite Geometry. Delft: Publications of the Delft Geodetic Computing Centre, LGR-Series, 1998. 18.

[26] Peng H M, Chang F R, Wang L S. Attitude Determination Using GPS Carrier Phase and Compass Data. Proceedings ION NTM-99, USA, Missouri, Kansas, 1999.727-732.

[27] Tiberius C C J M, de Jonge P J. Fast Positioning Using the LAMBDA Method. Proceedings DSNS-95, Norway, Bergen, 1995.8.

[28] Tiberius C C J M. Recursive Data Processing for Kinematic GPS Surveying. Delft: Publ Geodesy, Netherlands Geodetic Commission, 1998.45.

[29] Teunissen P J G. An Optimality Property of the Integer Least-Squares Estimator. Journal of Geodesy, 1999, 73: 587-593.

[30] Euler H J, Goad C. On Optimal Filtering of GPS Dual Frequency Observations without Using Orbit Information. Bull Geod, 1991, 65: 130-143.

[31] Gerdan G P. A Comparison of Four Methods of Weighting Double Difference Pseudo Range Measurements.Trans Tasman Surv, 1995, 1(1): 60-66.

[32] Gianniou M. Genauigkeitssteigerung Beikurzzeit-Statischen und Kinematischen Satellitenmessungenbishinzu Echtzeitanwendung[ Ph.D Thesis], Mnchen: DGK, Reihe C, 1996. 458.

[33] Jin X X, de Jong C D . Relationship between Satellite Elevation and Precision of GPS Code Measurements. Journal of Navigation, 1996, 49: 253-265.

[34] Tiberius C C J M, Teunissen P J G, de Jonge P J. Kinematic GPS: Performance and Quality Control. Proceedings KIS97, Canada, Banff, 1997. 289-299.

[35] Schaffrin B, Bock Y. A Unied Scheme for Processing GPS Dual-Band Observations. Bull Geod, 1988, 62:142-160.

[36] Bock Y. Medium Distance GPS Measurements. In: Teunissen P J G, Kleusberg A, Eds, GPS for Geodesy, 2nd Ed, New York: Springer-Verlag. 1998.

[37] Teunissen P J G. The Ionosphere-Weighted GPS Baseline Precision in Canonical Form. Journal of Geodesy, 1998, 72: 107-117. 